\title{
Traction-Separation Relationships for Hydrogen Induced Grain Boundary Embrittlement in Nickel via Molecular Dynamics Simulations
}

\author{
Wesley Barrows ${ }^{1}$, Rémi Dingreville ${ }^{2}$, Douglas Spearot ${ }^{1, *, \dagger}$ \\ ${ }^{1}$ Department of Mechanical Engineering, University of Arkansas, Fayetteville, AR \\ ${ }^{2}$ Sandia National Laboratories, Albuquerque, NM
}

\begin{abstract}
A statistical approach combined with molecular dynamics simulations is used to study the influence of hydrogen on intergranular decohesion. This methodology is applied to a Ni $\Sigma 3(112)[1 \overline{1} 0]$ symmetric tilt grain boundary. Hydrogenated grain boundaries with different $\mathrm{H}$ concentrations are constructed using an energy minimization technique with initial $\mathrm{H}$ atom positions guided by Monte Carlo simulation results. Decohesion behavior is assessed through extraction of a traction-separation relationship during steady-state crack propagation in a statistically meaningful approach, building upon prior work employing atomistic cohesive zone volume elements (CZVEs). A sensitivity analysis is performed on the numerical approach used to extract the traction-separation relationships, clarifying the role of CZVE size, threshold parameters necessary to differentiate elastic and decohesion responses, and the numerical averaging technique. Results show that increasing $\mathrm{H}$ coverage at the $\mathrm{Ni} \Sigma 3(112)[1 \overline{10}]$ grain boundary asymmetrically influences the crack tip velocity during propagation, leads to a general decrease in the work of separation required for crack propagation, and provides a reduction in the peak stress in the extracted traction-separation relationship. The present framework offers a meaningful vehicle to pass atomistically derived interfacial behavior to higher length scale formulations for intergranular fracture.
\end{abstract}

Keywords: Hydrogen embrittlement, grain boundaries, fracture, molecular dynamics

\footnotetext{
${ }^{*}$ Present Address: Department of Mechanical \& Aerospace Engineering, University of Florida, P.O. Box 116250, Gainesville, FL 32611.

† Corresponding Author: dspearot@ufl.edu, 352-392-6747
} 


\section{Introduction}

Embrittlement effects due to the uptake of atomic species from the environment (e.g. H, Cs, or I) are a well-known factor influencing stress corrosion cracking. Atomic species, such as $\mathrm{H}$ or I, can be transported easily by a combination of interstitial and intergranular diffusion [1-3], which in turn degrades the strength and ductility of the metal. However, the precise mechanisms by which hydrogen causes embrittlement have been the subject of considerable debate. Some of the proposed mechanisms include: (i) failure due to hydride formation [4,5], and in non-hydride forming metals (ii) hydrogen-enhanced localized plasticity (HELP) [6-8] and (iii) hydrogen-induced decohesion (HID) [9-11]. While researchers have successfully developed empirical continuum models for stress corrosion cracking [12-15], insufficient understanding persists regarding the fundamental coupled role of compositional and microstructural evolution at the atomic scale.

Hydrogen-induced decohesion, also known as hydrogen-enhanced decohesion (HEDE), refers to the reduction of the cohesive force and surface formation energy in the presence of hydrogen $[10,11]$. Although direct experimental evidence of the HID/HEDE mechanism has yet to be obtained [16], its role in hydrogen embrittlement is supported by observations of failure without significant plastic deformation [17], reduction of fracture strength [18], and by thermodynamic considerations $[19,20]$. The HELP mechanism is generally described as an increase in plasticity in a localized region ahead of a crack tip [8] resulting from an increase in dislocation nucleation and mobility and has been extensively studied [21-23]. Experimentally, HELP is justified by observations of localized ductile crack propagation rather than cleavage fracture ahead of a propagating crack tip [24] and it was shown through high resolution fractography of hydrogenated $\mathrm{Ni}$ that significant plasticity was localized to the fracture surfaces [22]. 
However, given the contradictory evidence presented in the literature, a consensus has yet to be reached on whether HELP or HID is the governing mechanism for hydrogen embrittlement, or to what extent both mechanisms contribute simultaneously to embrittlement. For example, in direct opposition to atomistic simulations indicating that the presence of $\mathrm{H}$ increases edge dislocation mobility in $\alpha$-Fe [25], Song and Curtin [26] showed that H leads to a resistance in dislocation motion. It is likely that no single mechanism conclusively accounts for premature failure due to hydrogen embrittlement and that the interplay of these mechanisms will further depend on microstructural factors, such as the local structure of the grain boundary or the lattice region ahead of the crack tip. These microstructural details can be ascertained from atomistic simulations.

For pristine grain boundaries, significant physical insight regarding the fracture process comes from atomistic studies of bicrystal grain boundaries with carefully chosen orientations of the applied load, crack front and lattice slip systems [27-30]. These simulations have helped clarify the effects of the bicrystallography, generalized stacking fault energies, temperature and other factors on dislocation nucleation, twinning and other processes occurring at the grain boundary crack tip. For example, Yamakov et al. [29] provided great details on the dynamics of intergranular crack propagation and different mechanisms of energy dissipation. One of the promising outcomes of the work of Yamakov et al. [29] and Spearot et al. [30] was the extraction of traction-separation relationships which can provide atomistic-based input for cohesive-zone models used in continuum fracture simulations [29,31-33].

For metallic systems with impurities, atomistic simulations including first principles methods have been used to study hydride precipitation, hydrogen segregation, and the embrittling potency of various elements [4,25,34-44]. For example, ab initio methods have been used to study energetic aspects of the interaction between $\mathrm{H}$ and defects in metallic materials including: (i) the reduction in 
cohesive energy due to the presence of $\mathrm{H}$ in $\mathrm{Ni}$ [38], $\mathrm{Al}$ and $\mathrm{Fe}$ [37], (ii) the strong binding energy between $\mathrm{H}$ and dislocation cores, which could prevent dislocation cross slip and promote planarity of slip in $\mathrm{Al}$ [39] and $\boldsymbol{\alpha}-\mathrm{Fe}$ [25], and (iii) the reduction in stacking fault energy in $\mathrm{Zr}[4,40]$, enabling enhanced plasticity as confirmed by larger scale atomistic simulations [4]. Interatomic potential based atomistic simulations have also been used to explore the role of hydrogen on embrittlement at larger length scales: (i) H lowered the stress intensity factor and energy required to generate new surfaces during crack propagation in single crystal $\mathrm{Ni}$ [45], (ii) low constant concentrations of $\mathrm{H}$ facilitated dislocation emission at a crack tip [41,42] and crack propagation [43], and (iii) Xu et al. [44] explored the effect of $\mathrm{H}$ concentration and location on the fracture mechanisms of single crystal $\mathrm{Ni}$ under tension and found that low $\mathrm{H}$ content facilitated crack propagation and created localized plasticity around the propagating crack tip. However, insufficient work has been done to explore embrittlement mechanisms in $\mathrm{Ni}$ via atomistic simulations for a realistic distribution of $\mathrm{H}$ across a representative grain boundary area, which is necessary to further elucidate the role of hydrogen on grain boundary embrittlement in ductile materials.

In the present work, atomistic simulations are performed to explore the influence of $\mathrm{H}$ impurities inserted at a $\mathrm{Ni} \Sigma 3(112)[1 \overline{1} 0]$ symmetric tilt grain boundary on decohesion mechanisms and strength during fracture. Although a single grain boundary is analyzed in this work, the algorithm presented can be implemented in a high-throughput fashion to analyze a wide range of grain boundary structures to elucidate the dependencies of HELP and HID embrittlement mechanisms on microstructural features. Section 2 provides details regarding the procedure used to build hydrogenated grain boundaries and to insert an intergranular crack of sufficient length for propagation. Decohesion is assessed through extraction of a traction-separation relationship during steady-state crack propagation in a statistically meaningful approach, building upon the work of Yamakov et al. [29] employing cohesive zone volume elements (CZVE) in the atomistic simulation. 
The first part of the results (Section 3) focuses on the influence of the numerical approach on the sensitivity of the extracted traction-separation relationships. The second part of the results (Section 4) discusses how $\mathrm{H}$ coverage influences the details of crack propagation along the $\Sigma 3(112)$ symmetric tilt grain boundary, including asymmetries depending on crack propagation direction, and the cohesive strength of this grain boundary.

\section{Steady-state crack propagation simulation model}

The simulation approach in this study is based on a molecular dynamics (MD) model of crack propagation under time-independent, or steady-state, conditions along a flat grain boundary. The approach is composed of three steps: (i) creation of a hydrogenated grain boundary, (ii) introduction and propagation of an atomically sharp crack, and (iii) data mining to extract the relationship between atomistic simulations and continuum interface separation laws. Steps (i) and (ii) are covered in this section whereas the data mining procedure is discussed in Section 3. All simulations in this work are performed using LAMMPS [46] with an embedded-atom method (EAM) potential for Ni$\mathrm{H}$ [47]. This EAM potential accurately predicts the bulk cohesive energy, elastic constants, the intrinsic stacking fault energy, and vacancy formation energy in $\mathrm{Ni}$. The $\mathrm{Ni}-\mathrm{H}$ interactions were fit to the solution energy and migration energy of $\mathrm{H}$ in Ni. This potential is not designed to capture hydride formation in the Ni-H system [47]; thus, analysis of this mechanism for embrittlement is beyond the scope of this work.

\subsection{Creation of hydrogenated grain boundaries}

Grain boundaries with $\Sigma 3$ misorientations are commonly observed in metallic materials that have been subjected to grain boundary engineering processing routes [48]; the lateral $\Sigma 3(112)$ [110] is selected in this work as it provides sufficient free volume for $\mathrm{H}$ segregation compared to the 
coherent $\Sigma 3$ twin grain boundary. The construction of a Ni $\Sigma 3(112)$ [110] symmetric tilt grain boundary with varying concentrations of $\mathrm{H}$ is done via a three-step process. First, the lowest energy hydrogen-free Ni $\Sigma 3(112)[1 \overline{1} 0]$ symmetric tilt grain boundary structure is determined using a relatively small bicrystal $(<15,000 \mathrm{Ni}$ atoms) with periodic boundary conditions. To determine the minimum energy configuration, one grain is incrementally displaced relative to the other, sampling $N_{\text {pos }}$ initial positions $\left(N_{p o s}=100\right)$ within the grain boundary plane, and subjected to energy

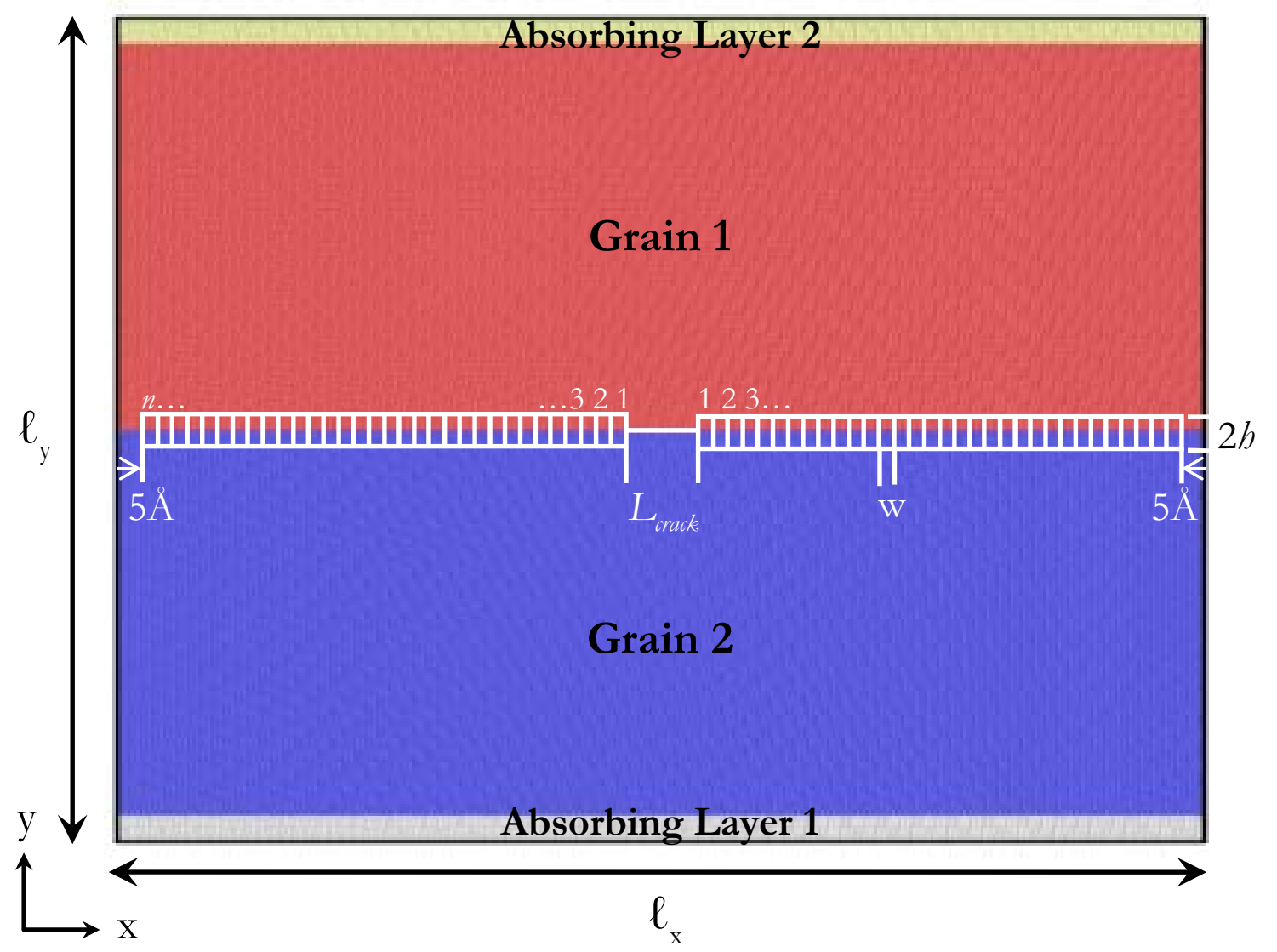

Figure 1. Schematic of the four layer crack-propagation model and cohesive zone volume element (CZVE) definition. The grain boundary between Grain 1 and Grain 2 is the primary grain boundary where the atomically sharp crack is introduced along with varying concentrations of hydrogen. The grain boundaries between Grain 1 and Absorbing Layer 2,

Grain 2 and Absorbing Layer 1, and Absorbing Layer 1 and 2 all serve to ensure that dislocations emitted during crack propagation do not pass across the periodic boundaries causing the crack to interact with its own periodic image. Atoms within $b$ of the primary grain boundary are assigned to CZVEs unless they fall within $5 \AA$ of the periodic boundary or within the region containing the atomically sharp crack, $L_{\text {crack }}$. 
minimization using the Polak-Ribiere version of the nonlinear conjugate gradient algorithm [49]. The translation corresponding to the minimum energy configuration is used to create larger systems utilized in the remainder of this study. The interface structure is consistent with prior work in the literature for the Ni $\Sigma 3(112)[1 \overline{1} 0]$ grain boundary [47].

Second, using the translation associated with the minimum energy $\Sigma 3(112)$ [110] grain boundary structure, larger simulation models with periodic boundary conditions are created that are comprised of four layers following the methodology proposed by Yamakov et al. [29], as shown in Fig. 1: Two primary grains, between which is the primary (hydrogenated) grain boundary, and two "absorbing layers" [29] (hydrogen-free) on either side of the primary grains. The boundaries between the absorbing layers and the primary grains as well as the grain boundary between the two absorbing layers across the periodic boundary are referred to as "absorbing grain boundaries" [29] and act simply as obstacles to prevent dislocations from passing through the periodic boundary. The dimensions of the atomistic system $\left(\ell_{\mathrm{x}}, \ell_{\mathrm{y}}\right.$, and $\left.\ell_{\mathrm{z}}\right)$ are chosen to be appropriate multiples of the grain boundary displacement shift complete distances [50], ensuring that no artificial boundaries or discontinuities are created as a result of the periodic boundary conditions. Specifically, for the lattice orientation in this manuscript, the simulation model $\ell_{\mathrm{x}}=914.523 \AA, \ell_{\mathrm{y}}=1102.309 \AA$ and $\ell_{\mathrm{z}}=$ $99.561 \AA$ is selected containing approximately 9.39 million atoms. This simulation model size is sufficient so that the crack can propagate more than five times its initial length without self-image interactions through the periodic boundaries.

In the final step of the construction process, hydrogen atoms are added at selected interstitial sites only within the primary grain boundary (between Grains 1 and 2), thus generating a hydrogenated model in which $\mathrm{H}$ has completely segregated to the grain boundary. These sites and the equilibrium 
concentration of $\mathrm{H}$ for the $\Sigma 3(112)[110]$ grain boundary are determined using Monte Carlo simulations performed at $0 \mathrm{~K}$ [51] with the same interatomic potential as the current work. The Monte Carlo simulations were performed for 100,000 steps per atom where $50 \%$ of the steps attempted to add or remove H. Figures 2 (c) and (d) show the three most energetically favorable locations for $\mathrm{H}$ within the $\Sigma 3(112)[1 \overline{10}$ ] grain boundary from Monte Carlo simulations [51]. These simulations also determined that the equilibrium $H$ concentration for the $\Sigma 3(112)[110]$ symmetric tilt grain boundary is approximately 300 appm based on a chemical potential $\mu=-2.35 \mathrm{eV}$ [51], which corresponds to a saturation grain boundary coverage of approximately $0.132 \mathrm{H} / \AA^{2}(66.4 \%$ of favorable sites are populated with $\mathrm{H}$ ). In the current work a range of $\mathrm{H}$ concentrations are studied: $100 \% \mathrm{H}$ saturation $\left(0.198 \mathrm{H} / \AA^{2}\right)$ refers to the configuration where every favorable site in each

(a)

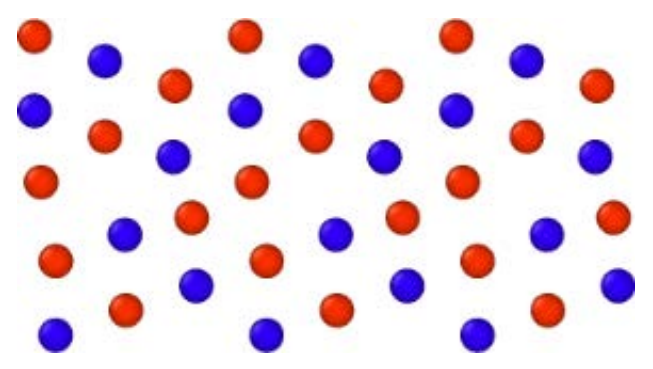

(c)

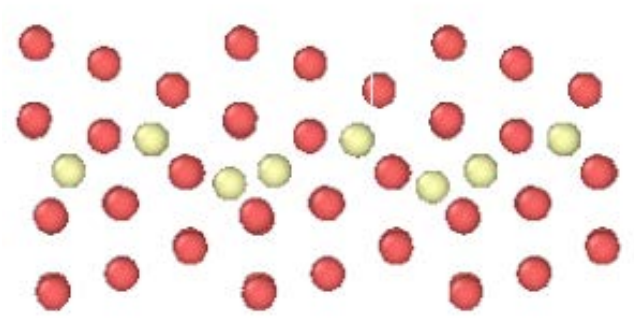

(b)

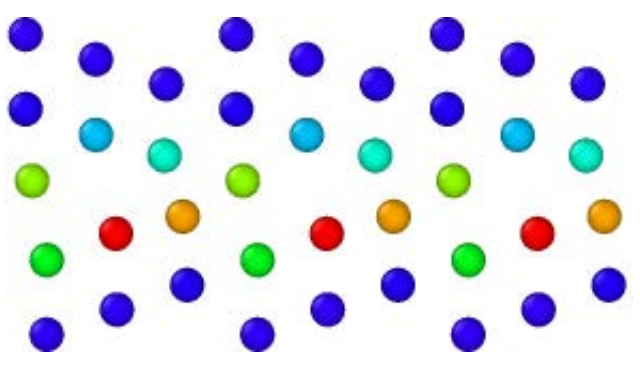

(d)

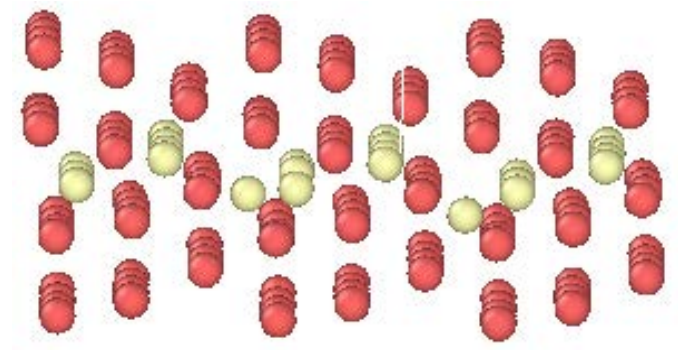

Figure 2. Structure of the primary $\Sigma 3(112[1 \overline{1} 0]$ grain boundary. (a) and (b) Hydrogen-free minimum energy grain boundary structures colored by $\{110\}$ atomic plane and the centrosymmetry parameter, respectively. (c) Projection along the misorientation axis and (d) rotated view of the grain boundary with $\mathrm{H}$ atoms (yellow) in the three most energetically favorable positions determined by Monte Carlo simulations [51]. 
structural unit at the grain boundary is occupied by a $\mathrm{H}$ atom; $75 \%\left(0.149 \mathrm{H} / \AA^{2}\right), 66.4 \%(0.132$ $\left.\mathrm{H} / \AA^{2}\right), 50 \%\left(0.099 \mathrm{H} / \AA^{2}\right)$, and $25 \%\left(0.050 \mathrm{H} / \AA^{2}\right)$ models are created by randomly removing $\mathrm{H}$ atoms from the fully populated $(100 \% \mathrm{H}$ saturation) grain boundary model. $0 \% \mathrm{H}$ refers to a pristine hydrogen-free $\mathrm{Ni}$ grain boundary. Thus, the $\mathrm{H}$ grain boundary coverages examined in this work span the equilibrium grain boundary coverage determined via Monte Carlo simulations.

\subsection{Steady-state crack propagation}

Once the hydrogenated grain boundary model is constructed with a desired H coverage, the fourlayer periodic grain boundary system is relaxed via energy minimization using the Polak-Ribiere version of the nonlinear conjugate gradient algorithm [49] for 5,000 iterations to allow the grain boundary structure to accommodate the added $\mathrm{H}$. Then, the temperature of the system is equilibrated to $300 \mathrm{~K}$ under a predefined hydrostatic tension $\sigma_{H}$ for $100,000 \mathrm{ps}$ in the isobaricisothermal (NPT) ensemble, reaching equilibrium at the specified temperature and prestress. As a result, the hydrogenated grain boundary model is strained in tension to provide a sufficient driving force for future crack propagation. In the case of the $\Sigma 3(112)[110]$ symmetric tilt grain boundary, $\sigma_{H}$ is set to $10 \mathrm{GPa}$ based on preliminary simulations with various crack lengths. Although local stresses near the grain boundary may be non-hydrostatic, dislocation emission is not observed under the prestress conditions. This general approach was initially developed to model steady-state crack propagation in pure Al by Yamakov et al. [29].

Once the system is equilibrated under the prestress conditions $\sigma_{H}$, the simulation is switched to an isovolume-isothermal (NVT) ensemble preserving the strain associated with the hydrostatic prestress. At this point, an atomically sharp crack of length $L_{c r a c k}$ is introduced in the center of the primary grain boundary, as shown in Fig. 1. The atomically sharp crack is introduced by screening the interactions between atoms in regions Grain 1 and Grain 2 only over the length $L_{\text {crack. }}$ The 
screening remains active throughout the simulation. For the $\Sigma 3(112)$ [110] symmetric tilt grain

boundary in this work, the screened region is set to $L_{\text {crack }}=50 \AA$ based on preliminary simulations to find a compatible combination of initial crack length and hydrostatic prestress appropriate for crack propagation. With the imposed strain due to the prestress $\sigma_{H}$ and initial crack $L_{\text {cracke }}$, the system is evolved for 40,000 ps which allows both crack tips (left and right directions) to propagate for approximately $100 \AA$ in each direction along the plane of the primary grain boundary. Simulations show that crack propagation and dislocation nucleation behavior are different for the left crack tip and the right crack tip. The role of $\mathrm{H}$ on this asymmetry will be examined in Section 4.

\section{Extraction of traction-separation behavior}

Cohesive traction-separation relationships are extracted from atomistic forces and displacements ahead of each crack tip by tracking the evolution of the state of the CZVEs along the grain boundary during steady-state crack propagation. This approach has been successfully applied to a pristine $\mathrm{Al} \Sigma 99$ bicrystal [29], results of which were reproduced to validate the implementation in this work. The influence of CZVE size, filtering approaches to deconvolute the elastic and decohesion response of the grain boundary, and the influence of different averaging techniques are explored in this section.

\subsection{Definition of cohesive zone volume elements (CZVE)}

Before the atomically sharp crack is introduced via the screening approach described in Section 2.2, each atom within a distance $b$ on either side of the primary grain boundary is assigned to a threedimensional rectangular volume element (called a Cohesive Zone Volume Element), as shown in Fig. 1. The state of each CZVE at position $x$ along the grain boundary and time $t$ is defined by two state variables: (i) the average normal stress, $\sigma_{y y}(x, t)$, computed using the virial stress definition [52], 
and (ii) the crack tip opening displacement, $\lambda(x, t)$. Thus, each CZVE state is represented as a point $\left(\lambda, \sigma_{y y}\right)$ in the $\sigma-\lambda$ configurational space at every snapshot in time. Using a statistical mechanics approach, in the limit of steady-state crack propagation that occurs over an infinitely long time over an infinitely long interface, all realized CZVE states will produce a density of states distribution $\rho(\lambda, \sigma)$ that is a continuous function independent of time. Via this approach, the decohesion behavior of the grain boundary is extracted as an average to mitigate the role of thermal vibrations and phonon wave propagation on the traction-separation relationship.

Specifically, the bounds of the CZVEs are determined by partitioning the prestressed system near the grain boundary into $4 n$ zones with $2 n$ zones above and $2 n$ zones below the primary grain boundary, resulting in $n$ CZVE pairs along the left and right crack propagation directions. A $5 \AA$ region near each periodic boundary is omitted in the assignment of cohesive zones; this does not affect the traction-separation results as the crack does not propagate to within $300 \AA$ of the periodic boundary. Also, cohesive zones above/below the initial $50 \AA$ crack are omitted and not used to determine the average traction-separation relationship as they are within the screening length. The crack tip opening displacement, $\lambda$, is computed by tracking the change in the y-position of the center of mass of two vertically neighboring cohesive zones (normal to the grain boundary plane) as the crack propagates. This work focuses only on normal displacement during crack propagation and does not consider tangential displacements. 

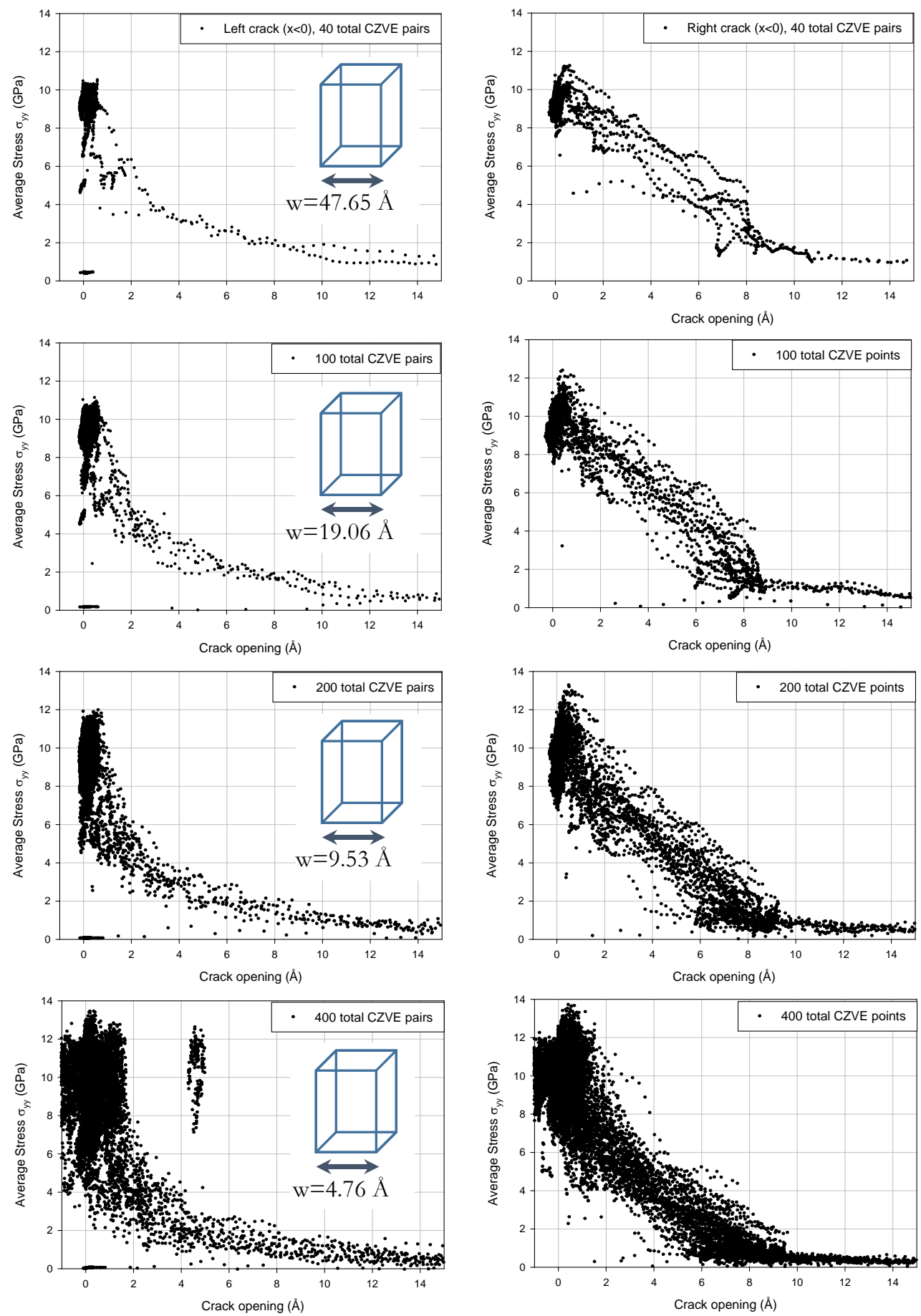

Figure 3. Traction-separation density of states, $\rho(\sigma, \lambda)$, for crack propagation to the left (left column) and right (right column) for a grain boundary with 25\% H using 40 (row 1), 100 (row 2), 200 (row 3), and 400 (row 4) total CZVE pairs. Using 40 and 100 CZVE pairs results in data sparsely populated in some crack opening bands while using 400 CZVE pairs results in a density highly dependent on thermal vibrations, phonon wave propagation, and dislocation nucleation events. 
A systematic survey was performed to determine the necessary size of each CZVE. Results, shown in Fig. 3, indicate that if the CZVEs are too small, the density of states $\rho(\lambda, \sigma)$ data is highly sensitive to thermal effects and dislocation activity due to the statistically insufficient number of atoms in each CZVE for averaging. Yet, if the CZVEs are too large, an insufficient number of $(\lambda, \sigma)$ data points are extracted resulting in improper statistics of the decohesion behavior of the crack tip. Thus, for the remainder of this work, 200 CZVE pairs (100 pairs on each side of the initial crack) with $w=9.53 \AA$ and $h=20 \AA$ and containing roughly 1,500 atoms in each CZVE are used to effectively mitigate thermal and phonon effects.

\subsection{Deconvolution of elastic and decohesion response}

As the crack unzips along the grain boundary plane, the raw density of states data includes information related to the decohesion response of the grain boundary, but also data associated with non-decohesion behavior such as the elastic response of the grain boundary to the hydrostatic prestress. The extracted traction-separation relationships should correspond uniquely to the decohesion behavior of the grain boundary without bias from the imposed prestress.

As shown in Fig. 4, the raw $(\lambda, \sigma)$ density of states represents four approximate regions corresponding to different positions of the CZVE pairs relative to the propagating crack tip. Region 1 roughly encompasses states corresponding to CZVE pairs sufficiently far away from the propagating crack tip to be unaffected or minimally affected by the plasticity ahead of the crack tip or the crack tip itself. Thus a majority of the states (low $\lambda$, high $\sigma$ ) in Region 1 correspond to the elastic stiffness of the grain boundary. Region 2 encompasses states corresponding to CZVE pairs sufficiently close to the crack tip to be affected by the plasticity generated during propagation or containing the crack tip. Regions 3 and 4 encompass states corresponding to CZVE pairs through which the crack tip has propagated and are distinguished by the degree of separation; states of 


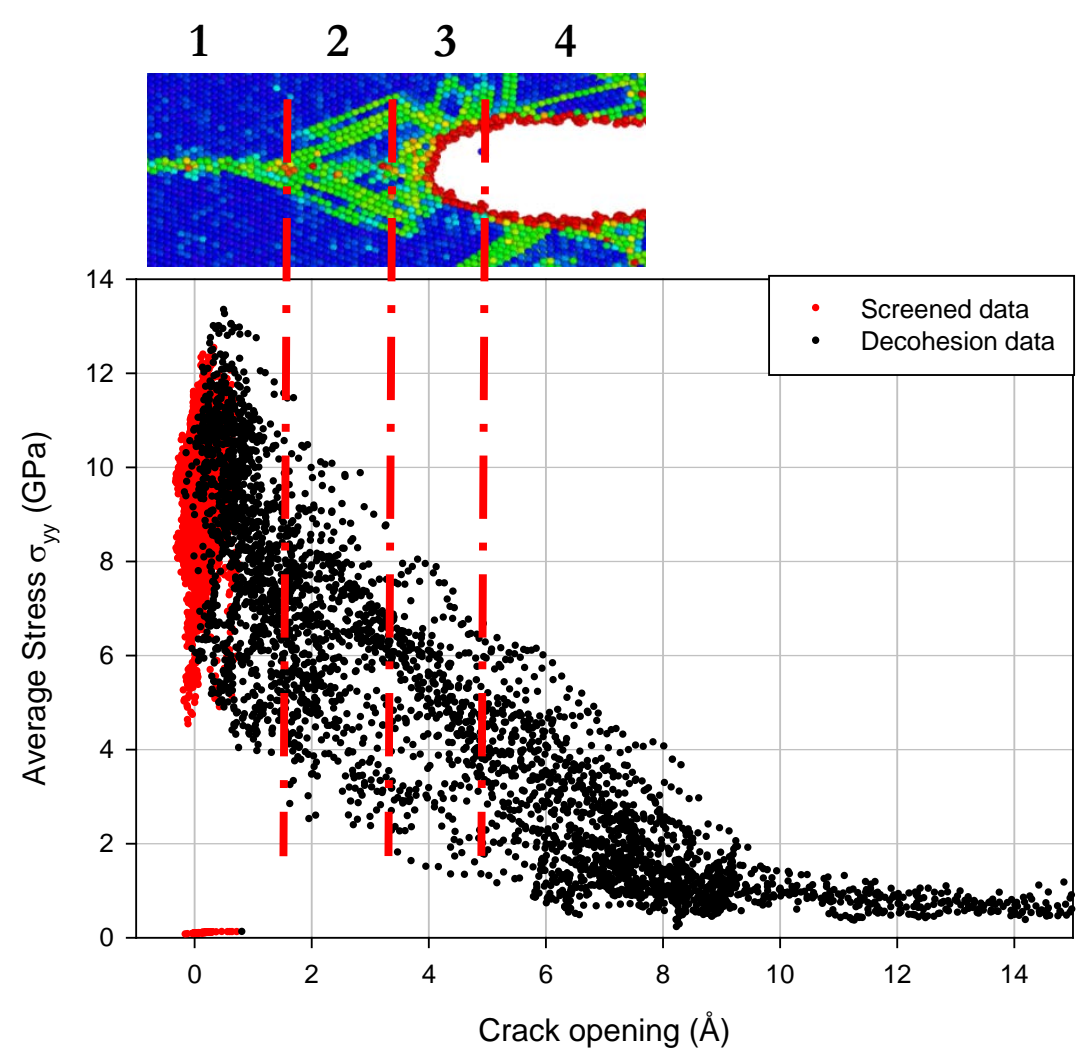

Figure 4. Anatomy of the traction-separation curve. Region 1 contains states corresponding to CZVE pairs far ahead of the crack tip to be unaffected by the plasticity ahead of the crack tip or the crack tip itself. Data points in Region 2 correspond to CZVE pairs significantly affected by the plasticity ahead of the crack tip or contain the crack tip. Data points in Region 3 correspond to separated CZVE pairs through which the crack tip has passed through but have not separated completely. Data points in Region 4 correspond to CZVE pairs far behind the crack tip that have significantly separated. The screened points, shown in red, are omitted when including $2 \mathrm{CZVE}$ ahead of the left crack tip with $25 \% \mathrm{H}$ coverage at the primary grain boundary.

CZVE pairs in Region 3 have separated significantly but are not fully separated whereas states of CZVE pairs in Region 4 have fully separated.

To limit the set of $(\lambda, \sigma)$ data to that exclusively associated with the decohesion response of the grain boundary, the density of states is filtered to include only CZVEs that are at or in the local neighborhood ahead of the propagating crack tip. To quantitatively track the position of the crack tip, a crack tip opening displacement threshold is defined, above which a cohesive zone pair is 
considered open, meaning that the crack is within or has propagated through this pair of cohesive zones. In this work, a cohesive zone pair is considered open if the normal opening displacement is greater than $\delta=1.5 \AA$. While this threshold could arguably be affected by the activities of defects ahead of the crack tip, in order to avoid any convoluted effect of an evolving normal opening displacement being dependent on the local plasticity, the threshold $\delta$ has been chosen to be constant and greater than the Burger's vector of a Shockley partial dislocation in Ni (1.437 $\AA$ ) and thus the CZVE pair would not be considered open after a single partial dislocation emission event.

(a)

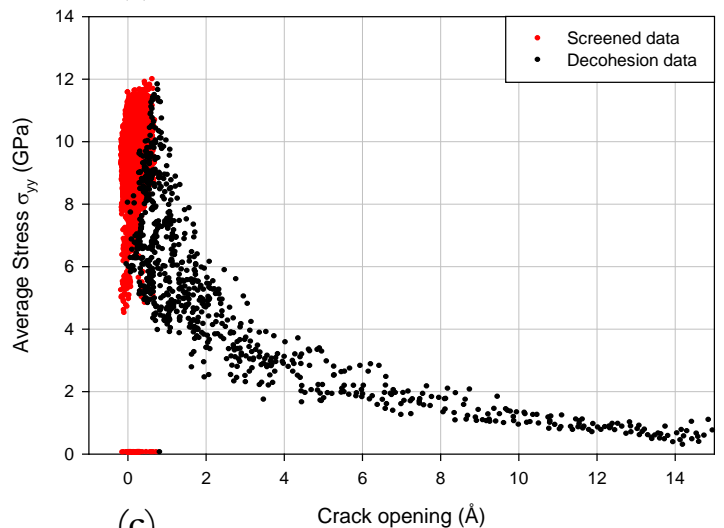

(c)

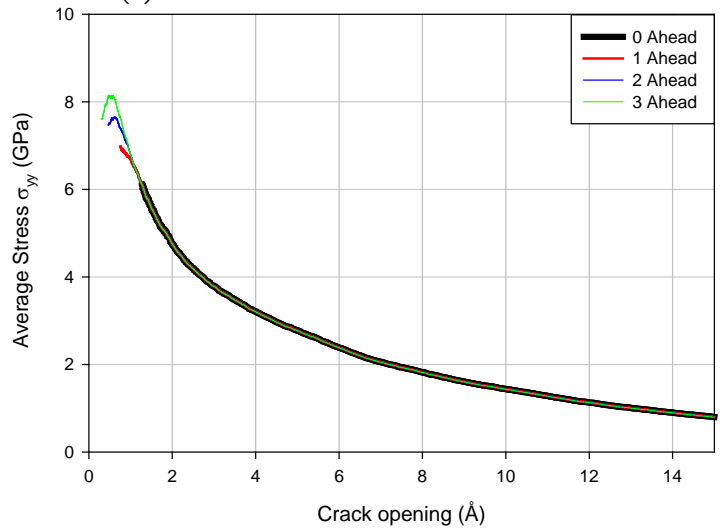

(b)

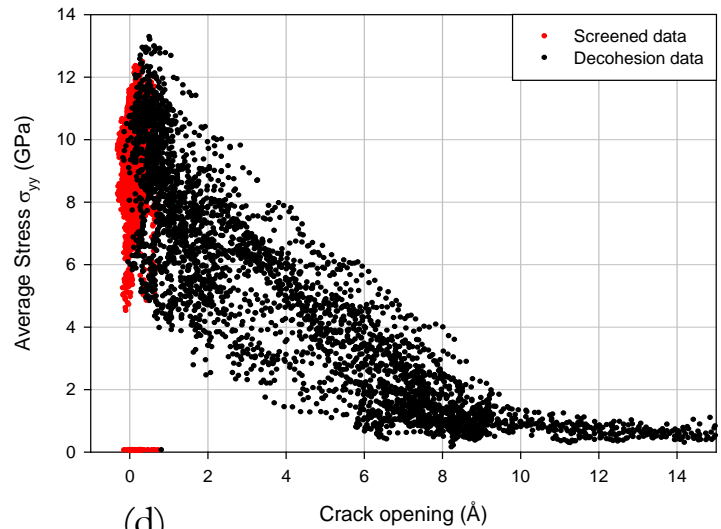

(d)

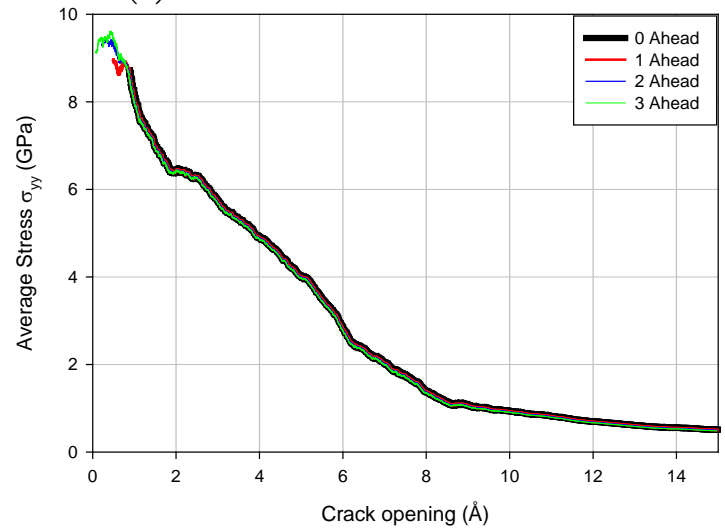

Figure 5. (a) and (b) Density of states for CZVEs located ahead of the left and right crack tips, respectively. Red data points correspond to screened elastic stiffness points when including 2 CZVE ahead of the propagating crack tips. (c) and (d) Survey of the effect of inclusion of different numbers of CZVEs ahead of propagating crack tip on the traction-separation relationship for both left (c) and right (d) crack tips for $25 \% \mathrm{H}$ coverage at the grain boundary. All simulations are performed using 100 CZVE pairs ahead of each crack tip and plots are generated using a running averaging with $M=250$ points. 
Effectively, by screening all data points with opening displacements less than the $1.5 \AA$ opening criteria, the final traction-separation relationship is composed of points only in Regions 2, 3, and 4 in Fig. 4. Figure 5 shows traction-separation relationships including $0,1,2$, and 3 CZVEs ahead of the crack tip (along with all opened CZVEs) in the density of states. In Figs. 5(c) and 5(d), a running average with $M=250$ data points is used to generate the traction-separation curve from the density of states data. Through this filtering approach, the $(\lambda, \sigma)$ data points for CZVE pairs far ahead of the crack tip, a majority of which remain at equilibrium at the hydrostatic prestress of $10 \mathrm{GPa}$ (states in Region 1 of Fig. 4), are excluded from the grain boundary decohesion relationship. Using this simple comparative sensitivity analysis, it is concluded that data from 2 CZVE pairs ahead of the crack tip should be utilized. The combined effect of only including data from open CZVE pairs and 2 unopened CZVE pairs ahead of the crack tip, while not including data from CZVE bordering free surfaces or the periodic boundary, ensures that the traction-separation relationship corresponds to the decohesion behavior of the grain boundary with limited effects of the grain boundary elastic stiffness or other physical mechanisms.

\subsection{Influence of averaging scheme on traction-separation}

The state of each cohesive zone volume element $\left(\lambda, \sigma_{\mathrm{yy}}\right)$ is computed at a constant time interval $\Delta \mathrm{t}=$ 250 ps during crack propagation. After generating the density of states, a curve fit is applied to the deconvoluted traction-separation data. As a part of this study, the results of two averaging techniques are analyzed to determine which method provides the best average representation of the density of states $\rho(\lambda, \sigma)$ over a wide range of crack tip opening displacements. The first averaging technique fits a piece-wise linear curve to the filtered traction-separation data by dividing the range of crack tip opening displacements into $N$ equally spaced bins, representing a small interval $(\lambda-\Delta \lambda$, $\lambda+\Delta \lambda$ ), over which the traction and crack tip opening displacements are averaged resulting in a 
single data point for every one of the $N$ bins. A survey of the effect of the averaging bin size on the traction-separation relationship is provided in Fig. 6(a) for propagation of the left crack tip. The second averaging technique fits a piece-wise linear curve to the filtered traction-separation data using a running average technique. Specifically, the first point in the running average is computed by averaging the first $M\left(\lambda, \sigma_{y y}\right)$ values. Each subsequent point in the running average is generated by shifting the range of $M$ points by 1 . A survey of the effect of the number of points $M$ employed in the running average on the traction-separation relationship is shown in Fig. 6(b) for propagation of the left crack tip.

(a)

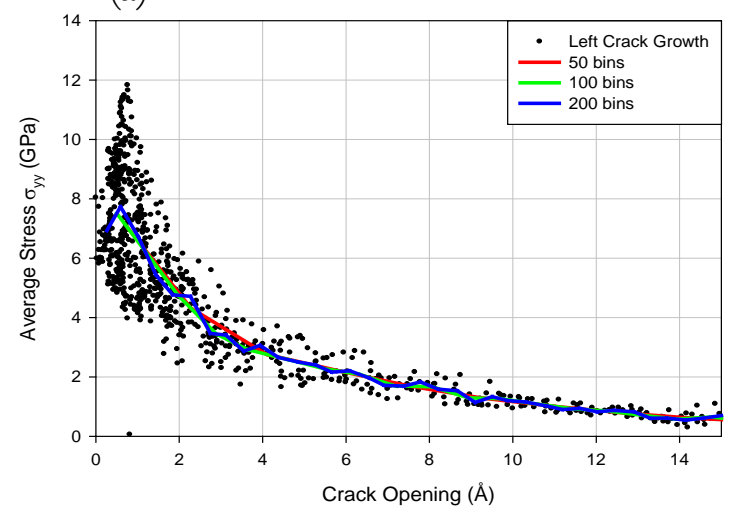

(b)

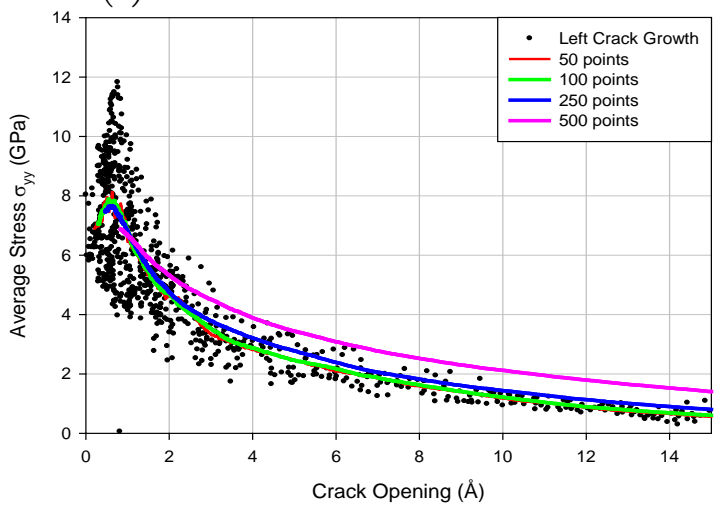

Figure 6. Survey of the effect of averaging methodology on the extracted traction-separation curves using filtered data for propagation of the left crack tip with $25 \% \mathrm{H}$ coverage: (a) survey of bin size effects (b) survey of running average parameter, $M$, effects.

Based on these results, the running average technique with $M=250$ is chosen as the most appropriate technique to extract a smooth traction-separation relationship while accurately capturing the peak and the shape of the density of states data. One of the limitations of the binning approach is that each bin contains an inconsistent number of data points; bins associated with small opening displacements represent many more CZVE states than bins associated with large opening displacements. Average points generated using the binning technique are highly sensitive to the 
number of points in the binning region. Alternatively, the running average technique is preferred over the binning average technique as it is able to capture a clear maximum in the tractionseparation relationship while representing a uniform number of CZVE states. Note, Fig. 6 shows data for crack propagation in the left direction, these conclusions are consistent for the analysis of crack propagation in the right direction for the $\Sigma 3(112)[110]$ grain boundary.

\section{Role of $\mathbf{H}$ on intergranular embrittlement}

The outlined numerical methodology in Sections 2 and 3 to extract the traction-separation relationship from $\rho(\sigma, \lambda)$ is applied to the $\Sigma 3(112)[110]$ grain boundary in $\mathrm{Ni}$ with systematically varying $\mathrm{H}$ coverage at the primary grain boundary to explore the effect of $\mathrm{H}$ coverage on the traction-separation relationship. Insights on plasticity and decohesion mechanisms in the vicinity of the crack tip and their relation with the work of separation for the $\Sigma 3(112)$ [110] symmetric tilt grain boundary are discussed in this section.

\subsection{Crack tip plasticity}

A qualitative analysis of left and right crack tips during steady-state crack propagation shows that varying the coverage of $\mathrm{H}$ at the grain boundary influences the structure of the grain boundary and dislocation emission from the crack tip. Figure 7 shows the atomistic region around the propagating crack after 10,000 ps with different coverage amounts of $\mathrm{H}$ at the primary grain boundary. Recall that each model is subjected to an identical initial hydrostatic pre-tension. First, it is clear that the structure of the grain boundary is modified by the presence of hydrogen. Indeed, in Fig. 7(a) the discrete extended stacking faults associated with the hydrogen free grain boundary structure in the vicinity of the crack tips are observable, while in Fig. $7(\mathrm{e})$ the $100 \%$ saturated grain boundary 
(a)

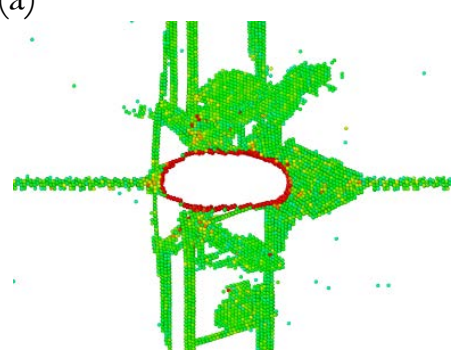

(d)

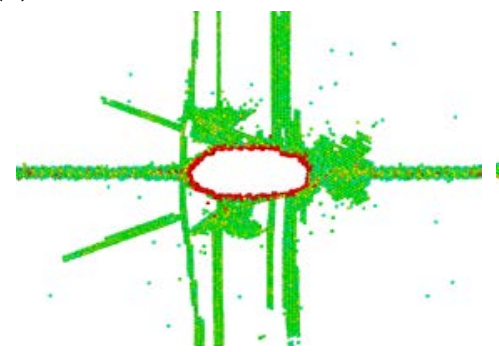

(b)

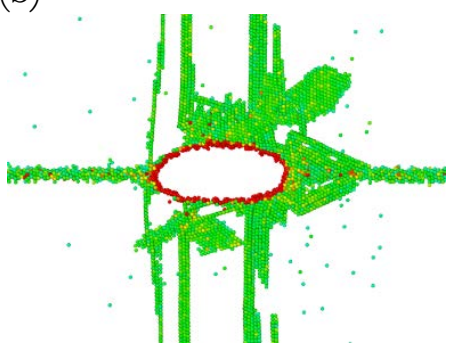

(e)

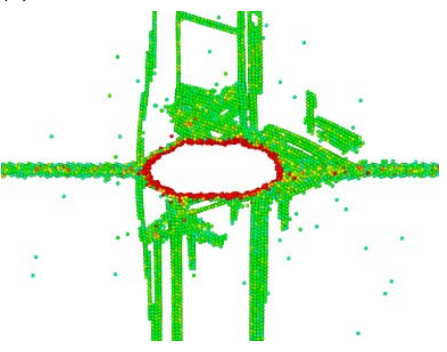

(c)

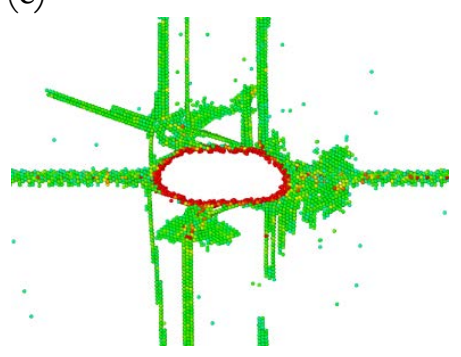

(f)

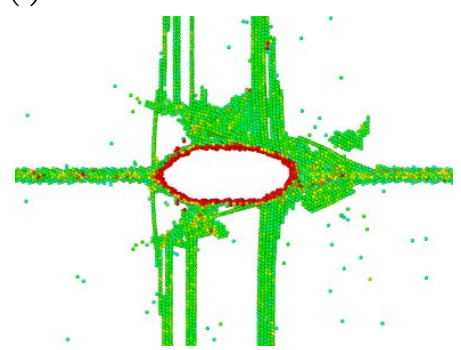

Figure 7. Dislocation activity during crack propagation colored by the centrosymmetry parameter at 10,000 ps with (a) $0 \%$, (b) $25 \%$, (c) $50 \%$, (d) $66.4 \%$, (e) $75 \%$, and (f) $100 \%$ H coverage at the primary grain boundary. Hydrogen saturation at the grain boundary influences both the structure of the grain boundary and dislocation nucleation during crack propagation.

structure identified via centrosymmetry is more uniform along the grain boundary plane with a finite thickness. Second, due to the crystallographic orientation of each lattice relative to the left and right crack tips, asymmetric dislocation nucleation occurs. The left crack tip emits the same twin dislocation in all systems whereas the density and type of dislocations nucleated from the right crack tip varies with $\mathrm{H}$ coverage. Figure 7 qualitatively shows that dislocation nucleation is at a maximum for steady-state crack propagation along the hydrogen-free grain boundary and at a minimum for either the $50 \% \mathrm{H}$ or $66.4 \% \mathrm{H}$ grain boundary model. This variability may be due to the randomness associated with the sites occupied by the $\mathrm{H}$ atoms added to the vicinity of the primary grain boundary. Recall, the $66.4 \% \mathrm{H}$ coverage corresponds to the equilibrium saturated state of the $\Sigma 3(112)[1 \overline{1} 0]$ grain boundary predicted from Monte Carlo calculations. 


\subsection{Decohesion behavior}

The quantitative effect of varying $H$ coverage at the primary $\Sigma 3(112)$ [110] symmetric tilt grain

boundary is discussed in terms of the crack tip velocity during propagation, the work of separation of the grain boundary, and the peak stress of the extracted traction-separation relationships.
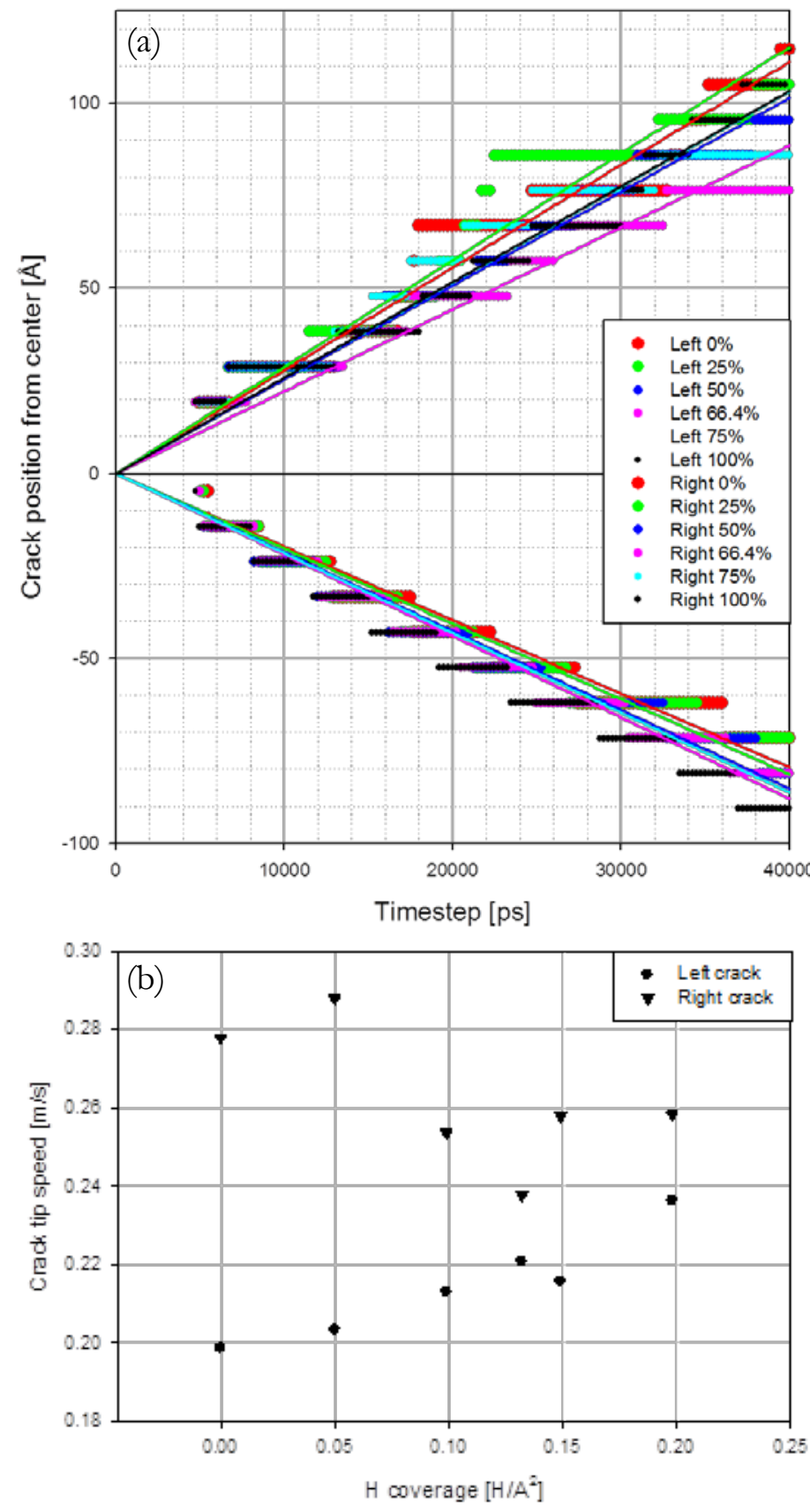

Figure 8. (a) Crack tip position as a function of time. Propagation of the right crack tip is positive and propagation of the left crack tip is negative relative to the grain boundary model coordinate system, The data for $66.4 \%$ coverage corresponds to the simulation shown in Fig. 7.(b) Crack tip speed as a function of $\mathrm{H}$ coverage. The left and right crack tip speeds for the $66.4 \%$ coverage case are the average values of the three simulations that were performed. 
First regarding the crack tip velocity, recall, the crack tip position and associated velocity are identified by tracking the center of mass position of vertically neighboring pairs of CZVEs along the grain boundary. As shown in Fig. 8, the crack tip velocity, computed by linearly interpolating the crack tip position data as a function of time, shows asymmetry depending on the crack propagation direction (velocities listed in Table 1). As illustrated in Fig. 8(b), when propagating to the left (-x direction), the crack tip velocity generally increases with increasing $\mathrm{H}$ concentration. However, when propagating to the right ( $+\mathrm{x}$ direction), the crack tip velocity generally decreases with increasing $\mathrm{H}$ concentration. Interestingly, the crack tip velocities are converging towards each other as $\mathrm{H}$ coverage is increased at the primary grain boundary. This is rationalized by examining the structure of the grain boundary ahead of each crack tip, as shown in Fig. 7. As observed in Section 4.1, hydrogen promotes a more uniformly distorted grain boundary structure, mitigating both the influence of the orientation of the extended stacking faults characteristic of the $\Sigma 3(112)$ [110] grain boundary (Fig. 7(a)) and the orientation of the $\Sigma 3(112)[110]$ structural units relative to the activated slip systems in each lattice. Finally, note that both left and right crack tip velocities are reasonably
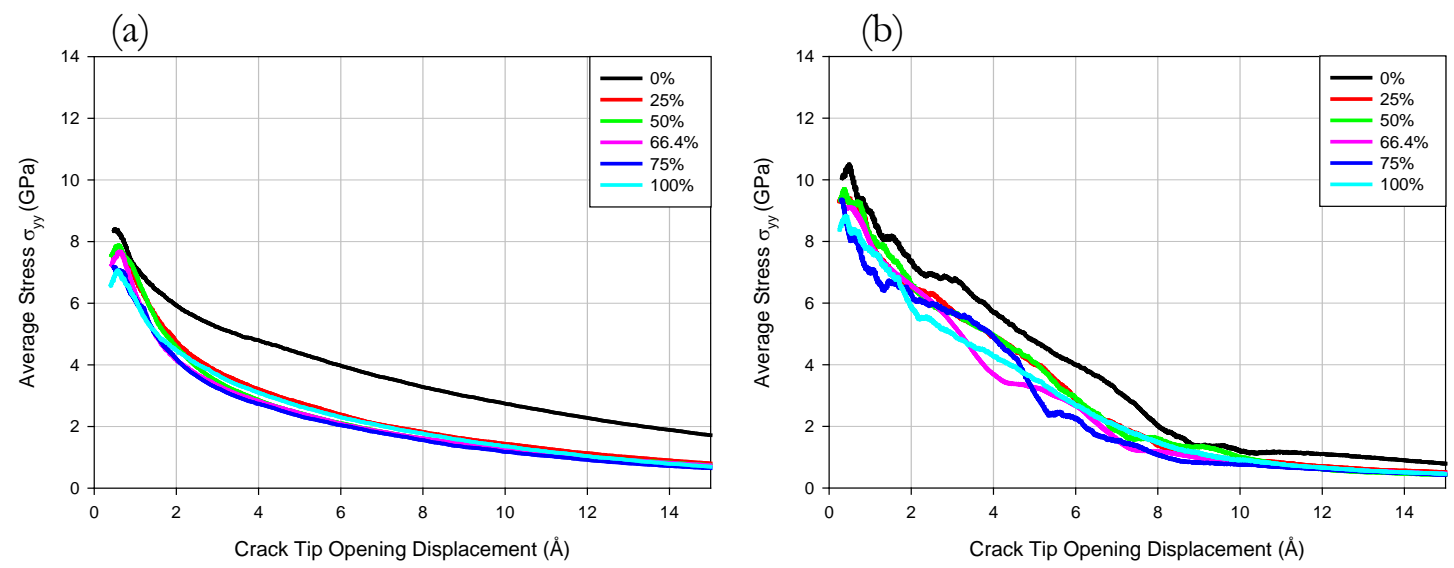

Figure 9. Role of $\mathrm{H}$ saturation on traction-separation curves of (a) left (-x direction) and (b) right (+x direction) crack tips. All simulations are performed using 100 CZVE ahead of each crack tip and plots generated using running average technique with $M=250$ points. The plots for the $66.4 \%$ H coverage case correspond to the simulation shown in Fig. 7. 
(a)

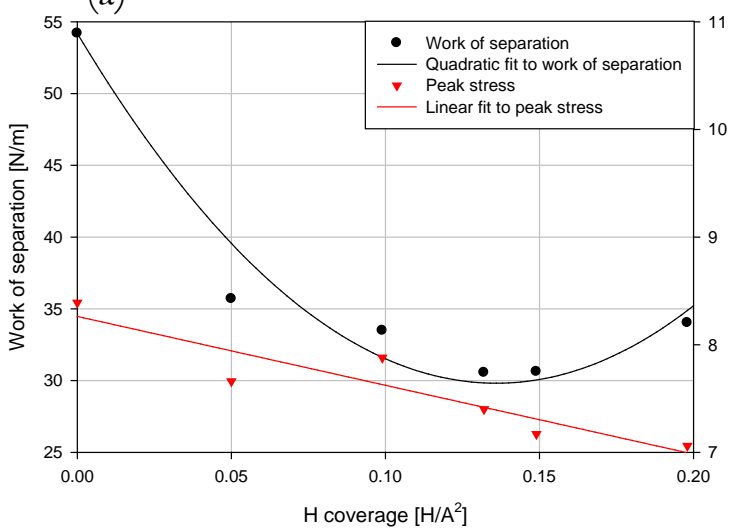

(b)

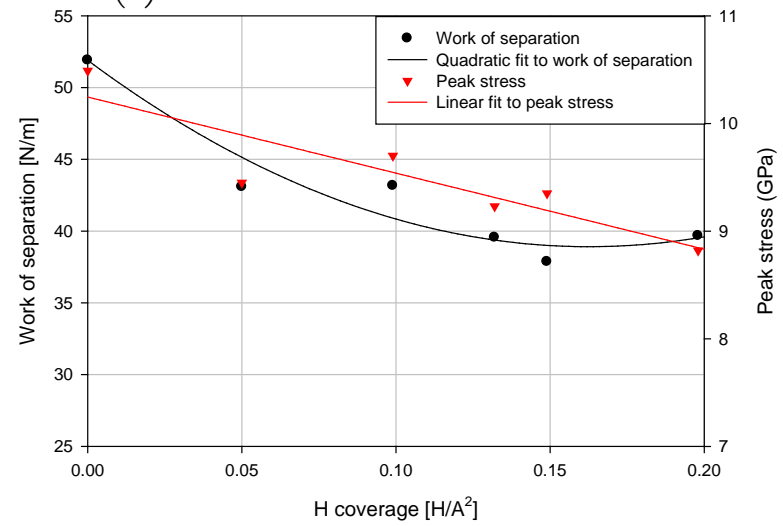

Figure 10. Dependence of the work of separation and peak stress as a function of $\mathrm{H}$ coverage for propagation of the (a) left (-x direction) and (b) right ( $+x$ direction) crack tips. The values for peak stress and work of separation for both the left and right crack tips for the $66.4 \%$ coverage case are the average values of the three simulations that were performed. Quadratic and linear fits are provided to illustrate general trends and not meant to indicate a specific mechanism.

constant over the simulation time, supporting the assumption of steady-state crack propagation necessary for extracting decohesion relationships.

Second, as illustrated in Figs. 9 and 10, the peak stress for the $\Sigma 3(112)[110]$ symmetric tilt grain boundary shows a general decrease with increasing $\mathrm{H}$ coverage at the primary grain boundary (data provided in Table 1). An exception to this is the half-saturated $(50 \% \mathrm{H})$ grain boundary which shows a slight increase in the peak stress relative to the $25 \% \mathrm{H}$ saturated grain boundary.

Finally, the work of separation (the work required to separate the grain boundary into two free surfaces) can be computed by integrating the traction-displacement curve (a maximum opening displacement of $15 \AA$ is selected for this integration). As shown in Fig. 10, the work of separation for the $\Sigma 3(112)[1 \overline{10}]$ symmetric tilt grain boundary generally decreases with increasing $\mathrm{H}$ saturation until a minimum is reached around the equilibrium $\mathrm{H}$ saturation of $66.4 \% \mathrm{H}$ coverage. Then, the work of separation modestly increases for the $100 \% \mathrm{H}$ coverage which is severely oversaturated compared to equilibrium determined by Monte Carlo calculations. 


\begin{tabular}{|c|c|c|c|c|c|c|}
\hline & $\begin{array}{c}0 \% \mathbf{H} \\
\text { Saturation }\end{array}$ & $\begin{array}{c}25 \% \mathrm{H} \\
\text { Saturation }\end{array}$ & $\begin{array}{c}50 \% \mathrm{H} \\
\text { Saturation }\end{array}$ & $\begin{array}{c}66.4 \% \mathrm{H} \\
\text { Saturation }\end{array}$ & $\begin{array}{c}75 \% \mathrm{H} \\
\text { Saturation }\end{array}$ & $\begin{array}{c}100 \% \mathrm{H} \\
\text { Saturation }\end{array}$ \\
\hline & $\begin{array}{c}0.000 \mathrm{H} / \\
\AA^{2}\end{array}$ & $\begin{array}{c}0.050 \mathrm{H} / \\
\AA^{2}\end{array}$ & $\begin{array}{c}0.099 \mathrm{H} / \\
\AA^{2}\end{array}$ & $\begin{array}{c}0.132 \mathrm{H} / \\
\AA^{2}\end{array}$ & $\begin{array}{c}0.149 \mathrm{H} / \\
\AA^{2}\end{array}$ & $\begin{array}{c}0.198 \mathrm{H} / \\
\AA^{2}\end{array}$ \\
\hline \multicolumn{7}{|c|}{ Left Crack Tip } \\
\hline $\begin{array}{c}\text { Crack Tip } \\
\text { Velocity } \\
{[\mathrm{m} / \mathrm{s}]}\end{array}$ & 0.1987 & 0.2034 & 0.2131 & $\begin{array}{c}0.2208 \pm \\
0.007\end{array}$ & 0.2157 & 0.2364 \\
\hline $\begin{array}{c}\text { Peak Stress } \\
{[\mathrm{GPa}]}\end{array}$ & 8.39 & 7.66 & 7.88 & $7.40 \pm 0.27$ & 7.17 & 7.06 \\
\hline $\begin{array}{c}\text { Work of } \\
\text { Separation } \\
{[\mathrm{N} / \mathrm{m}]}\end{array}$ & 54.19 & 35.69 & 33.48 & $\begin{array}{c}30.55 \pm \\
2.239\end{array}$ & 30.62 & 34.02 \\
\hline \multicolumn{7}{|c|}{ Right Crack Tip } \\
\hline $\begin{array}{c}\text { Crack Tip } \\
\text { Velocity } \\
{[\mathrm{m} / \mathrm{s}]}\end{array}$ & 0.2779 & 0.2879 & 0.2536 & $\begin{array}{c}0.2378 \pm \\
0.0257\end{array}$ & 0.2579 & 0.2583 \\
\hline $\begin{array}{c}\text { Peak Stress } \\
{[\mathrm{GPa}]}\end{array}$ & 10.49 & 9.45 & 9.70 & $9.23 \pm 0.13$ & 9.35 & 8.82 \\
\hline $\begin{array}{l}\text { Work of } \\
\text { Separation } \\
{[\mathrm{N} / \mathrm{m}]}\end{array}$ & 51.90 & 43.08 & 43.16 & $\begin{array}{c}39.55 \pm \\
1.552\end{array}$ & 37.86 & 39.67 \\
\hline
\end{tabular}

Table 1. Crack tip velocity and work of separation for both crack tips at all saturation levels simulated. Crack tip velocity is determined by fitting a line to crack tip propagation data; peak stress is determined using $M=250$ running average technique to extract traction-separation relationship; work of separation is computed using a numerical integration technique.

It is recognized that statistical variability exists within molecular dynamics simulations. To assess the extent of this variability, three simulations with different random realizations of $\mathrm{H}$ coverage at the grain boundary and different random initialized thermal velocities have been conducted. The standard deviations associated with the computed values for peak stress and work of separation are presented in Table 1 and show that statistical variability has very little impact on the observed trends for peak stress and work of separation as a function of $H$ coverage in the $\Sigma 3(112)[110]$ grain boundary.

Although only one grain boundary is examined in this work, with the primary focus on the numerical implementation of algorithms to model steady-state crack propagation and to capture grain boundary decohesion via atomistic CZVEs, aspects consistent with both HID and HELP can 
be identified in the separation response. The left crack tip nucleates the same twinning dislocation during crack propagation, regardless of $\mathrm{H}$ saturation; yet, the velocity of the crack increases and the work of separation and peak strength decrease with increasing $\mathrm{H}$ coverage. These observations are consistent with the theory of the HID mechanism, and are consistent with prior results for low $\mathrm{H}$ coverages [43]. Plasticity nucleating from the right crack tip appears to decrease as $\mathrm{H}$ coverage increases from $0 \% \mathrm{H}$ to $66.4 \% \mathrm{H}$ (equilibrium saturation) and then begins to increase as $\mathrm{H}$ coverage increases from $66.4 \% \mathrm{H}$ to $100 \% \mathrm{H}$, with different slip systems activated at different concentrations of $\mathrm{H}$ (Fig. 7). Thus, for this propagation direction both HID and HELP may be appropriate to describe crack propagation depending on $\mathrm{H}$ saturation. A more detailed analysis of the dislocation emission processes from the $\Sigma 3(112)[110]$ grain boundary is necessary, combined with a CZVE analysis of a wide range of grain boundaries, before quantitative conclusions can be reached regarding the contribution of HID and HELP theories and their relationship to grain boundary structure.

\section{Conclusions}

In this work, a methodology is employed to extract traction-separation relationships from molecular dynamics simulations of crack propagation along hydrogenated $\Sigma 3(112)$ [110] grain boundaries. Sensitivity of the extracted traction-separation relationship to the numerical approach is extensively studied. This methodology is then used to explore the effects of varying $\mathrm{H}$ coverage along the $\mathrm{Ni}$ $\Sigma 3(112)[110]$ grain boundary on the decohesion mechanisms, as assessed through the tractionseparation relationships. Four main conclusions can be made as a result of this work:

1. The traction-separation relationship is dependent on the size of the CVZEs, the number of CZVE that are included in the density of states, and the numerical averaging technique used to derive the cohesive law from $\rho(\sigma, \lambda)$. 
2. For the $\Sigma 3(112)[110]$ symmetric tilt grain boundary, the velocity of the left (-x) crack tip generally increases with increasing $\mathrm{H}$ coverage while the velocity of the right $(+\mathrm{x})$ crack tip generally decreases. Such a trend can be associated with a change in the grain boundary structure due to the $\mathrm{H}$ coverage and the role of slip system orientation.

3. For crack propagation in either direction, the work of separation generally decreases with increasing $\mathrm{H}$ saturation until a minimum is reached around the equilibrium $\mathrm{H}$ saturation (in this specific illustration around $66.4 \% \mathrm{H}$ coverage). Then, the work of separation modestly increases for the oversaturated $\mathrm{H}$ coverage.

4. The peak stress during decohesion decreases with increasing $\mathrm{H}$ concentration (with the exception of the $50 \%$ saturation for the particular primary grain boundary studied). The peak stress in all systems with $\mathrm{H}$ is less than the pure $\mathrm{Ni}$ system by between $0.51-1.33 \mathrm{GPa}$ for propagation of the left crack tip and $0.79-1.67 \mathrm{GPa}$ for propagation of the right crack tip.

Future work will continue to elucidate the embrittlement mechanisms caused by $\mathrm{H}$ in $\mathrm{Ni}$ using the outlined methodology to extract the traction-separation relationships from other grain boundary systems. As shown in this work, the interplay between hydrogen-induced mechanisms such as HID and HELP, and the structure of the grain boundary relative to the lattice crystallography is a critical aspect of hydrogen embrittlement that is currently unresolved. Future work will also attempt to characterize the defects nucleating from the crack tips and relate the defects to the embrittlement mechanisms experienced. Ultimately, information gleaned from atomistic studies may be used in higher length scale formulations for intergranular fracture [31,53,54] by augmenting the classical framework with a cohesive law model and a set of internal state variables representing the grain boundary state. 


\section{Acknowledgements}

Supported by the Laboratory Directed Research and Development program at Sandia National Laboratories, a multi-program laboratory managed and operated by Sandia Corporation, a wholly owned subsidiary of Lockheed Martin Corporation, for the U.S. Department of Energy's National Nuclear Security Administration under contract DE-AC04-94AL85000. Simulations were performance on the Arkansas High Performance Computing Center, supported in part by the National Science Foundation under grants MRI \#0722625, MRI-R2 \#0959124, and \#0918970. 


\section{References}

[1] W.S. Ryu, Y.H. Kang, J.-Y. Lee, J. Nucl. Mater. 152 (1988) 194-203.

[2] a. Pundt, R. Kirchheim, Annu. Rev. Mater. Res. 36 (2006) 555-608.

[3] R.K. Dayal, N. Parvathavarthini, Sadhana 28 (2003) 431-451.

[4] M. Ruda, G. Bertolino, D. Farkas, A. Baruj, Comput. Mater. Sci. 69 (2013) 327-334.

[5] H.G. Nelson, Metall. Trans. Phys. Metall. Mater. Sci. 7 (1976) 621-627.

[6] H.K. Birnbaum, P. Sofronis, Mater. Sci. Eng. A 176 (1994) 191-202.

[7] P. Novak, R. Yuan, B.P. Somerday, P. Sofronis, R.O. Ritchie, J. Mech. Phys. Solids 58 (2010) 206-226.

[8] C.D. Beachem, Metall. Trans. 3 (1972) 437-451.

[9] A.R. Troiano, Met. Prog. 77 (1960) 112-117.

[10] E.A. Steigerwald, F.W. Schaller, A.R. Troiano, Metall. Soc. Am. Inst. Mining, Metall. Pet. Eng. - Trans. 218 (1960) 832-841.

[11] R.A. Oriani, P.H. Josephic, Scr. Metall. 8 (1974) xii.

[12] K. Sieradzki, R.C. Newman, J. Phys. Chem. Solids 48 (1987) 1101-1113.

[13] Y.S. Garud, Corrosion 46 (1990) 968-974.

[14] L.-O. Jernkvist, in:, Trans. 12th Int. Conf. Struct. Mech. React. Technol., Publ by Elsevier Science Publishers B.V, Stuttgart, Germany, 1993, p. 75.

[15] R. Falkenberg, W. Brocks, W. Dietzel, I. Schneider, Key Eng. Mater. 417-418 (2009) 329_ 332.

[16] P. Sofronis, I.M. Robertson, AIP Conf. Proc. 837 (2006) 64-70.

[17] J. Kameda, C.J. McMahon Jr., Metall. Trans. Phys. Metall. Mater. Sci. 14 A (1983) 903-911.

[18] M. Dadfarnia, B.P. Somerday, P.E. Schembri, P. Sofronis, J.W. Foulk, K. a. Nibur, D.K. Balch, Jom 66 (2014).

[19] J.P. Hirth, Metall. Trans. Phys. Metall. Mater. Sci. 11 (1980) 861-890.

[20] Y. Mishin, P. Sofronis, J.L. Bassani, Acta Mater. 50 (2002) 3609-3622. 
[21] D.F. Teter, I.M. Robertson, H.K. Birnbaum, Acta Mater. 49 (2001) 4313-4323.

[22] T. Matsumoto, J. Eastman, H.K. Birnbaum, Scr. Metall. 15 (1981) 1033-1037.

[23] S.P. Lynch, Acta Metall. 36 (1988) 2639-2661.

[24] J. Eastman, T. Matsumoto, N. Narita, F. Heubaum, H.K. Birnbaum, in:, Hydrog. Eff. Met. Proc. 3rd Int. Conf. Eff. Hydrog. Behav. Mater., Metall Soc of AIME, Moran, WY, USA, 1981, pp. 397-409.

[25] S. Taketomi, R. Matsumoto, N. Miyazaki, J. Mater. Sci. 43 (2008) 1166-1169.

[26] J. Song, W.A. Curtin, Acta Mater. 68 (2014) 61-69.

[27] F. Cleri, S.R. Phillpot, D. Wolf, Interface Sci. 7 (1999) 45-55.

[28] D. Farkas, Philos. Mag. Lett. 80 (2000) 229-237.

[29] V. Yamakov, E. Saether, E.H. Glaessgen, J. Mater. Sci. 43 (2008) 7488-7494.

[30] D.E. Spearot, K.I. Jacob, D.L. McDowell, Mech. Mater. 36 (2004) 825-847.

[31] V.R. Coffman, J.P. Sethna, G. Heber, M. Liu, A. Ingraffea, N.P. Bailey, E.I. Barker, Model. Simul. Mater. Sci. Eng. 065008 (2008) 18.

[32] J.T. Lloyd, J.A. Zimmerman, R.E. Jones, X.W. Zhou, D.L. McDowell, Model. Simul. Mater. Sci. Eng. 19 (2011) 065007.

[33] J. Zhai, V. Tomar, M. Zhou, J. Eng. Mater. Technol. 126 (2004) 179.

[34] P. Gumbsch, Mater. Sci. Eng. A 260 (1999) 72-79.

[35] M.L. Rossi, Open J. Phys. Chem. 01 (2011) 104-108.

[36] C.D. Taylor, Int. J. Corros. 2012 (2012).

[37] D.E. Jiang, E.A. Carter, Acta Mater. 52 (2004) 4801-4807.

[38] W. Geng, A. Freeman, R. Wu, C. Geller, J. Raynolds, Phys. Rev. B 60 (1999) 7149-7155.

[39] G. Lu, Q. Zhang, N. Kioussis, E. Kaxiras, Phys. Rev. Lett. 87 (2001) 095501.

[40] C. Domain, R. Besson, A. Legris, Acta Mater. 52 (2004) 1495-1502.

[41] M.S. Daw, M.I. Baskes, C.L. Bisson, W.G. Wolfer, in:, Model. Environ. Eff. Crack Growth Process. Proc. a Symp. Held 1985 Fall Meet. Metall. Soc., Metallurgical Soc of AIME, Sandia Natl Lab, Livermore, CA, USA, Sandia Natl Lab, Livermore, CA, USA, 1986, pp. 99-124. 
[42] Z. Li, J. Li, W. Chu, H. Liu, L. Qiao, J. Univ. Sci. Technol. Beijing Miner. Metall. Mater. (Eng Ed) 9 (2002) 59-64.

[43] R.G. Hoagland, H.L. Heinisch, J. Mater. Res. 7 (1992) 2080-2088.

[44] X. Xu, M. Wen, S. Fukuyama, K. Yokogawa, Mater. Trans. 42 (2001) 2283-2289.

[45] K.N. Solanki, D.K. Ward, D.J. Bammann, in:, Metall. Mater. Trans. A Phys. Metall. Mater. Sci., 2011, pp. 340-347.

[46] S. Plimpton, J. Comput. Phys. 117 (1995) 1-19.

[47] J.E. Angelo, N.R. Moody, M.I. Baskes, 3 (1995) 289-307.

[48] M. Seita, J.P. Hanson, S. Gradečak, M.J. Demkowicz, Nat. Commun. 6 (2015) 6164.

[49] E. Polak, G. Ribiere, ESAIM Math. Model. Numer. Anal. - Modélisation Mathématique Anal. Numérique 3 (1969) 35-43.

[50] W. Bollmann, Crystal Defects and Crystalline Interfaces, Springer, 1970.

[51] C.J. O’Brien, S.M. Foiles, Private Communication, 2014.

[52] D.H. Tsai, J. Chem. Phys. 70 (1979) 1375.

[53] A. Musienko, G. Cailletaud, Acta Mater. 57 (2009) 3840-3855.

[54] J.J. Rimoli, M. Ortiz, Philos. Mag. 90 (2010) 2939-2963. 